\title{
AVALIAÇÃO ECONÔMICA DE UM POVOAMENTO DE Eucalyptus grandis DESTINADO A MULTIPRODUTOS ${ }^{1}$
}

\author{
Thelma Shirlen Soares ${ }^{2}$, Rosa Maria Miranda Armond Carvalho² e Antonio Bartolomeu do Vale ${ }^{3}$
}

\begin{abstract}
RESUMO - O objetivo deste estudo foi realizar a avaliação econômica da utilização de multiprodutos em um povoamento de Eucalyptus grandis através dos métodos do VPL, TIR, BPE e B/C. Foram consideradas duas opções de utilização das toras de madeira: produção para um único uso e produção para multiprodutos. Na análise de sensibilidade foram consideradas variações na taxa de desconto e no custo da terra. A partir dos resultados obtidos foi possível concluir que a destinação do povoamento a multiprodutos foi a opção que apresentou maior viabilidade econômica em todas as situações estudadas.
\end{abstract}

Palavras-chave: Madeira para serraria, madeira para geração de energia e economia florestal.

\section{ECONOMIC EVALUATION OF Eeucalyptus grandis STANDS FOR MULTIPRODUCT USE}

\begin{abstract}
The objective of this study was to conduct an economic evaluation of the multiproducts in Eucalyptus grandis stands through the methods VPL, TIR, BPE and B/C. Two wood use alternatives were considered: single use and multiproducts productions. The sensitivity analysis included variations in the discount rate and land cost. The results showed that the multiproducts alternative presented higher economic feasibility in all the situations examined.
\end{abstract}

Key words: Saw timber, fuel wood and forest economics.

\section{INTRODUÇÃO}

Os projetos de reflorestamento no Brasil tiveram início com a introdução do eucalipto em 1904. Atualmente o setor florestal brasileiro mantém cerca de 4,8 milhões de hectares de florestas plantadas de rápido crescimento, distribuídos em todo o território nacional. Deste total, cerca de 3 milhões de hectares correspondem a plantações de eucalipto e 1,8 milhão de hectares a plantações de pinus (Floresta Brasil, 2001).

A madeira oriunda de plantios florestais é utilizada principalmente para produção de chapas, lâminas, compensados, aglomerados, carvão vegetal, madeira serrada, celulose e móveis. Dos plantios florestais podem ser obtidos outros produtos, como resinas, óleos essenciais e medicamentos, além de colaborarem para o seqüestro de carbono e contribuírem para a conservação das florestas naturais.

O setor industrial de base florestal tem sido marcado por um processo de utilização crescente de madeiras provenientes de reflorestamento, o que coloca o Brasil em sintonia com a ordem mundial, que enfatiza a preservação das florestas naturais e incentiva a implantação de florestas renováveis. O eucalipto apresenta-se como grande alternativa para produção de madeira nos próximos anos, e a indústria já aposta na sua disponibilidade

1 Recebido para publicação em 7.5.2002.

Aceito para publicação em 9.9.2003.

2 Doutoranda em Ciência Florestal na Universidade Federal de Viçosa - UFV, 36570-000 Viçosa-MG; ${ }^{3}$ Professor-Associado do Departamento de Engenharia Florestal - UFV. 
para os futuros suprimentos de matéria-prima. O descompasso crescente entre oferta e demanda de madeira nos mercados interno e externo tenderá a favorecer o quadro de substituição das madeiras nativas pela madeira de eucalipto (Castro, 2001).

Entretanto, verifica-se, mundialmente, o acirramento da concorrência, o que faz com que as empresas adotem uma nova mentalidade, que lhes propicie condições de crescimento, pois a busca pela formação de vantagens competitivas é um dos meios para garantir sua sobrevivência a longo prazo.

Sendo assim, pode-se afirmar que a multiplicidade de produtos florestais possíveis de se obter a partir dos povoamentos de eucalipto permite à empresa florestal direcionar suas atividades para o fornecimento de multiprodutos obtidos a partir da conversão da tora da madeira de eucalipto. Tal procedimento representa um diferencial competitivo, pois agrega uma crescente flexibilidade no que diz respeito à comercialização dos produtos de determinada empresa, propiciando a redução dos riscos de prejuízos.

Para Leite (1994) e Ferreira (1999), as receitas de um dado produto, que em determinado momento apresentar menor valor de mercado, poderão ser compensadas pelas receitas de outros produtos, de maior valor de venda, no momento de sua comercialização. Assim, a empresa florestal poderá otimizar e maximizar seus produtos, de forma a obter o máximo rendimento possível, que será transformado em lucro no final do processo

Para demonstrar que quando se enfoca um povoamento de eucalipto para fornecimento de mais de um produto florestal (multiprodutos) pode-se obter uma maior rentabilidade, desenvolveu-se o presente trabalho, cujo objetivo foi comparar a rentabilidade quando se explora o povoamento florestal para produção de um único produto versus a produção florestal para multiprodutos.

\section{MATERIAL E MÉTODOS}

\subsection{Caracterização dos Projetos}

Os dados utilizados foram obtidos do inventário florestal realizado em um povoamento de Eucalyptus grandis W.Hill ex Maiden, com 7 anos de idade, em espaçamento de $3 \times 2 \mathrm{~m}$, localizado na região sul mineira.

Tendo em vista determinar a utilização mais rentável da madeira de tal povoamento, realizou-se uma avaliação econômica considerando dois projetos (A e B).

O projeto A destina a produção a um único uso, ou seja, madeira para geração de energia, enquanto o projeto $\mathrm{B}$ destina a produção para multiprodutos, ou seja, madeira para serraria e madeira para geração de energia.

As especificações de cada tipo de tora relacionada ao uso da madeira estão no Quadro 1. A alternativa de mercado 1 refere-se à venda de madeira para serraria e a alternativa de mercado 2 refere-se à venda de madeira destinada à geração de energia.

Quadro 1 - Especificações ou características da madeira para serraria e energia

Table 1 - Specifications or characteristics of saw timber and fuel wood

\begin{tabular}{|c|c|c|}
\hline \multirow{2}{*}{$\begin{array}{c}\text { Alternativa de } \\
\text { Mercado }\end{array}$} & Diâmetro & Comprimento da tora \\
\cline { 2 - 3 } & $\geq 10 \mathrm{~cm}$ & $2,2 \mathrm{~m}$ \\
\hline 1 & $\geq 5 \mathrm{~cm} \mathrm{e}<10 \mathrm{~cm}$ & $2,2 \mathrm{~m}$ \\
\hline 2 &
\end{tabular}

\subsection{Estimativas da Produtividade}

A estimativa da produção foi obtida através do ajuste de modelos volumétricos e de razão de volume. Em todas as situações a seleção do modelo mais eficiente foi baseada no maior coeficiente de determinação $\left(\mathrm{R}^{2}\right)$, no menor erro-padrão residual (Syx) e na melhor distribuição gráfica de resíduos.

A produção volumétrica total do povoamento foi obtida através da função logarítmica de Spurr, cuja equação ajustada foi

$$
\ln V=-10,070451+0,972565 \ln \left(d a p^{2} \mathrm{x} H T\right)
$$

$V=$ volume total com casca $\left(\mathrm{m}^{3}\right) ;$ dap = diâmetro medido a $1,30 \mathrm{~m}$ de altura $(\mathrm{cm}) ; H=$ altura total $(\mathrm{m})$; e $\ln =$ logaritmo neperiano.

Já a produção comercial foi obtida através do modelo de razão volumétrica de Amateis e Burkhart para estimar o volume até qualquer diâmetro superior, obtendo-se a seguinte equação após o ajuste:

$$
V c=V\left[1-0,314398 \frac{(H-h)^{2,588321}}{H^{2,183942}}\right]
$$


em que

$V c=$ volume comercial com casca, até a altura comercial estabelecida $\left(\mathrm{m}^{3}\right) ; V=$ volume total com casca $\left(\mathrm{m}^{3}\right) ; d=$ diâmetro mínimo comercial estabelecido $(\mathrm{cm})$; dap = diâmetro medido a $1,30 \mathrm{~m}$ de altura $(\mathrm{cm}) ; h=$ altura comercial (m); e $H=$ altura total (m).

Os volumes comerciais totais estimados para o povoamento, nas diferentes opções de utilização da madeira, estão no Quadro 2.

Quadro 2 - Produção volumétrica dos projetos A e B Table 2 - Volume production of the projects $A$ and $B$

\begin{tabular}{|c|c|c|}
\hline Projeto & Alternativas de Mercado & $\begin{array}{c}\text { Volume Comercial } \\
\left(\mathrm{m}^{3} / \mathrm{ha}\right)\end{array}$ \\
\hline A & 2 & 231,74 \\
\hline \multirow{2}{*}{ B } & 1 & 178,49 \\
\cline { 2 - 3 } & 2 & 53,25 \\
\hline
\end{tabular}

\subsection{Custos e Receitas}

Para permitir a avaliação econômica dos projetos, foram obtidas informações a respeito dos custos e das receitas das alternativas de comercialização da madeira para os usos definidos nos projetos A e B.

Os custos referentes às operações de implantação, manutenção anual e exploração florestal, bem como o valor de mercado de cada produto, estão apresentados nos Quadros 3 e 4. Como custo da terra considerou-se o valor de US\$ 450,00/ha. Neste estudo, considerou-se o transporte da madeira como de responsabilidade do comprador.

\subsection{Critérios de Avaliação Financeira}

Após a obtenção do volume de cada produto, por hectare, procedeu-se à análise financeira dos projetos, considerando uma taxa de desconto de $10 \%$ ao ano.

Os critérios de análise econômica de projetos empregados foram: valor presente líquido $(V P L)$, taxa interna de retorno $(T I R)$, benefício periódico equivalente $(B P E)$, razão benefício-custo $(B / C)$ e valor esperado da terra $(V E T)$.
Quadro 3 - Itens de custo dos projetos A e B

Table 3 - Costs components of the projects $A$ and $B$

\begin{tabular}{|l|c|c|c|}
\hline \multirow{2}{*}{ Atividade } & \multirow{2}{*}{$\begin{array}{c}\text { Ano de } \\
\text { ocorrência }\end{array}$} & \multicolumn{2}{|c|}{ Custos (US\$/ha)* } \\
\cline { 3 - 4 } & & Projeto A & Projeto B \\
\hline Implantação ${ }^{\frac{1}{}}$ & 0 & 450,00 & 450,00 \\
\hline Capina & 1 & 45,00 & 45,00 \\
\hline Capina & 2 & 18,00 & 18,00 \\
\hline Manutenção & de 1 a 7 & 10,00 & 10,00 \\
\hline Exploração $^{2 /}$ & 7 & 3,50 & 4,00 \\
\hline
\end{tabular}

1/ Os custos de implantação envolvem as operações de infra-estrutura, construção de estradas e aceiros, desmatamento, destoca, limpeza da área, preparo do terreno, plantio e replantio de mudas, combate às formigas e aplicação de fertilizantes. ${ }^{2 /}$ Custos expressos em US $\$ / \mathrm{m}^{3}$.

* Dados fornecidos pelos produtores da região sul do Estado de Minas Gerais.

Quadro 4 - Preços de venda das toras de madeira* Table 4 - Log sale prices

\begin{tabular}{|c|c|}
\hline Alternativa de Mercado & $\mathrm{US} \$ / \mathrm{m}^{3}$ \\
\hline 1 & 13,20 \\
2 & 5,78 \\
\hline
\end{tabular}

* Preços da madeira explorada.

Segundo Rezende \& Oliveira (1993), esses métodos consideram o valor do capital no tempo, ou seja, atribuem diferentes ponderações às receitas líquidas em função de sua distribuição ao longo do tempo, sendo os mais indicados para a análise de projetos no setor florestal.

$$
\begin{aligned}
& V P L=\sum_{j=1(1+1)^{j}}^{n}-\sum_{j=1}^{n} \frac{C_{j}}{(1+1)^{j}} \\
& T I R \Rightarrow \sum_{j=1}^{n} \frac{R_{j}}{(1+T I R)^{j}}-\sum_{j=1}^{n} \frac{C_{j}}{(1+T I R)^{j}}=0 \\
& B P E=\frac{\left.V P L\left[(1+i)^{t}-1\right] 1+i\right)^{n t}}{(1+i)^{n t}-1} \\
& B / C=\frac{V B(i)}{V C(i)}
\end{aligned}
$$

em que $V P L=$ valor presente líquido no início do período $0 ; B P E=$ benefício periódico equivalente; $B / C=$ razão

R. Árvore, Viçosa-MG, v.27, n.5, p.689-694, 2003 
benefício-custo à taxa de descontos $i ; C M P=$ custo para produzir uma unidade adicional do produto; $T I R=$ taxa interna de retorno; $V B(i)=$ valor presente à taxa $i$ da seqüência de benefícios; $V C(i)=$ valor presente, à taxa $i$, dos custos do projeto; $R j=$ receitas no período $j ; C j=$ custos no período $j ; i=$ taxa de desconto; $j=$ período de ocorrência de $R j$ e $C j ; i=$ taxa de desconto; $n=$ duração do projeto (em anos); e $t=$ número de períodos de capitalização.

\subsection{Análise de Sensibilidade}

$\mathrm{Na}$ análise de sensibilidade, utilizou-se apenas o valor presente líquido (VPL) para verificar a viabilidade econômica dos projetos. Desta forma, realizou-se uma análise de sensibilidade do VPL às variações nas taxas de desconto e no custo da terra, para estabelecer a influência destes fatores na viabilidade econômica dos projetos. Também foi realizada a análise de sensibilidade, considerando, para uma taxa de $10 \%$ ao ano, a possibilidade de substituição da destinação da madeira do projeto A, ou seja, considerou-se que o projeto A seria destinado à venda de madeira para serraria.

Com relação às taxas de desconto, além da taxa de $10 \%$ ao ano utilizada inicialmente, considerou-se a aplicação de taxas reais de desconto de 6, 9 e $12 \%$ ao ano, no cálculo do VPL. Essas taxas foram escolhidas por (Oliveira et al., 1998) abrangerem os níveis mais usados pelo setor florestal.

\section{RESULTADOS E DISCUSSÃO}

\subsection{Avaliação da Viabilidade Econômica}

Um projeto é considerado viável economicamente se apresentar $V P L>0, T I R>$ taxa mínima de atratividade (TMA), BPE > 0, B/C>1 e VET > custo da terra. Desta forma, analisando o Quadro 5 é possível verificar que, a uma taxa de desconto de $10 \%$ ao ano, todos os critérios de avaliação econômica apontam o projeto A como inviável e o projeto $\mathrm{B}$ como viável do ponto de vista econômico. Tal fato indica que a alternativa de uso da madeira para geração de energia não é lucrativa, no referente caso, em relação ao uso de madeira para multiprodutos (serraria + energia), devido à maior valorização da tora para multiprodutos (serraria + energia). Portanto, destinar a produção a um único uso poderá resultar em prejuízo em relação ao uso de multiprodutos.

R. Árvore, Viçosa-MG, v.27, n.5, p.689-694, 2003
Quadro 5 - Valor presente líquido (VPL), taxa interna de retorno $(T I R)$, benefício periódico equivalente $(B P E)$ e razão benefício/custo $(B / C)$ dos projetos $A$ e $B$ para uma taxa de desconto de $10 \%$ a.a.

Table 5 - Net present value (VPL), internal rate of return (TIR), equivalent period value (BPE) and benefit/cost ratio $(B / C)$ of projects $A$ and $B$ for a discount rate of 10\% a.a.

\begin{tabular}{|c|c|c|c|c|}
\hline Projeto & $\begin{array}{c}\text { VPL } \\
\text { (US\$/ha) }\end{array}$ & TIR (\%) & $\begin{array}{c}\text { BPE } \\
\text { (US\$/ha) }\end{array}$ & B/C \\
\hline A & $-502,41$ & $-10,31$ & $-103,20$ & 0,58 \\
B & 117,76 & 12,77 & 24,19 & 1,09 \\
\hline
\end{tabular}

\subsection{Análise de Sensibilidade}

\subsubsection{Efeito da Taxa de Desconto sobre a Viabilidade Econômica}

O Quadro 6 apresenta o efeito da taxa de desconto sobre o VPL para os dois projetos analisados. Nota-se que a uma da taxa de desconto de $12 \%$ ao ano todos os projetos foram inviáveis economicamente. Analisando o projeto $\mathrm{A}$, observa-se que à medida que a taxa de desconto aumenta o risco do empreendimento também é maior e o $V P L$ cresce em termos negativos, ou seja, o prejuízo aumenta. O projeto $B$, por sua vez, também apresenta decréscimos do valor do $V P L$ à medida que a taxa de desconto aumenta. Esta sensibilidade às variações na taxa real de desconto usada era esperada, uma vez que altas taxas de desconto tendem a inviabilizar investimentos a longo prazo, devido ao longo tempo de conversão do investimento, como é o caso de investimentos no setor florestal. Neste contexto, sugere-se o uso de taxas inferiores àquelas usadas por outros setores da economia, a fim de viabilizar economicamente os projetos.

Quadro 6 - Valor presente líquido (VPL) para os projetos $A$ e $B$, considerando taxas de desconto $(i)$ de 6,9 e $12 \%$ a.a.

Table 6 - Net present value (VPL) for projects $A$ and $B$, based on discount rates (i) of $6 \%, 9 \%$ and $12 \%$ a.a.

\begin{tabular}{|c|c|c|}
\hline \multirow{2}{*}{$i$} & \multicolumn{2}{|c|}{$V P L$ (US\$/ha) } \\
\cline { 2 - 3 } & Projeto A & Projeto B \\
\hline 6\% a.a. & $-363,63$ & 440,12 \\
9\% a.a. & $-471,57$ & 189,55 \\
$12 \%$ a.a. & $-557,60$ & $-10,92$ \\
\hline
\end{tabular}




\subsubsection{Efeito do Preço da Terra sobre a Viabilidade Econômica}

O Quadro 7 mostra o VPL para a taxa de desconto de $10 \%$ ao ano e os níveis de variação do preço da terra entre $\pm 10 \%$ e $\pm 20 \%$. O projeto A, em todas as situações, apresentou VPL com valores negativos, indicando a inviabilidade econômica da produção de madeira para energia. Para o projeto B, observa-se que aumentos na variação do preço da terra implicam decréscimos no valor do VPL, o que indica que aumentos no custo da terra afetam bastante o VPL.

Quadro 7 - Valor presente líquido $(V P L)$ para variações porcentuais no custo da terra dos projetos $A$ e $B$, considerando a taxa de desconto $(i)$ de $10 \%$ a.a.

Table 7 - Net present value (VPL) for percentage variations in land cost of projects $A$ and $B$, based on a discount rate (i) of $10 \%$ a.a.

\begin{tabular}{|c|c|c|}
\hline Variação no custo da terra & \multicolumn{2}{|c|}{$V P L$ (US\$/ha) } \\
\cline { 2 - 3 }$(\%)$ & Projeto A & Projeto A \\
\hline-20 & $-458,60$ & 161,58 \\
-10 & $-480,51$ & 139,67 \\
+10 & $-524,32$ & 95,85 \\
+20 & $-546,23$ & 73,95 \\
\hline
\end{tabular}

\subsubsection{Efeito da Mudança de Opção de Produto do Projeto A sobre a Viabilidade Econômica}

Utilizando os dados de custos e receitas apresentados anteriormente, realizou-se a análise econômica, considerando para o projeto A a opção de venda de madeira destinada à serraria, em vez de madeira para energia. O projeto B permaneceu inalterado. Foi considerado, portanto, que o projeto A teria uma produção de $178,4926 \mathrm{~m}^{3}$ de madeira destinada para serraria.

Analisando o Quadro 8, verifica-se que para uma taxa de $10 \%$ ao ano o UPL apresenta-se positivo para ambos os projetos analisados. O projeto B apresenta-se ligeiramente superior ao projeto $\mathrm{A}$, indicando ser a utilização do povoamento para multiprodutos a opção mais viável economicamente. Entretanto, considerando a mudança de destinação do projeto A, pode-se observar claramente que a venda de madeira para serraria é a opção mais lucrativa quando comparada com a venda de madeira somente para energia, cujo VPL encontrado
(Quadro 5) foi de -US\$502,41/ha. Tal fato é explicado pela maior valorização das toras destinadas à serraria. Essa análise indica que, conforme apresentado por Silva et al. (1999), quando se utiliza a madeira para fins mais nobres viabilizam-se, em muito, os projetos florestais.

Quadro 8 - Valor presente líquido $(V P L)$ para os projetos $A$ e $B$, considerando a taxa de desconto $(i)$ de $10 \%$ a.a.

Table 8 - Net present value (VPL) for projects $A$ and $B$, based on a discount rate (i) of $10 \%$ a.a.

\begin{tabular}{|c|c|}
\hline Projeto A & Projeto B \\
\hline \multicolumn{3}{|c|}{$V P L(\mathrm{US} \$ / \mathrm{ha})$} \\
\hline 114,92 & 117,76 \\
\hline
\end{tabular}

\section{CONCLUSÕES}

Para as condições em que se desenvolveu este estudo, pode-se concluir que:

- Dentre os projetos avaliados, o projeto B foi o que apresentou melhor resultado em todas as situações, comprovando a maior rentabilidade econômica da destinação de um povoamento para multiprodutos.

- Aumentos na taxa de desconto afetam significativamente o VPL, indicando que quanto menor a taxa de desconto utilizada maior será a lucratividade do projeto.

- Aumentos no custo da terra também afetam significativamente a rentabilidade dos projetos, devendo ser ressaltado que quanto mais alto for o custo da terra, para ambos os projetos analisados, menor será a lucratividade obtida.

\section{REFERÊNCIAS BIBLIOGRÁFICAS}

CASTRO, J. A madeira de eucalipto. Disponível em: <http://www.floresta.ufpr.br/eventos/eucalipto/artigos.htm>. Acesso em: 25 julho 2001.

FERREIRA, S. O. Estudo da forma do fuste de Eucalyptus grandis e Eucalyptus cloeziana. 1999. 132 f. Dissertação (Mestrado em Engenharia Florestal) Universidade Federal de Lavras, Lavras, 1999.

FLORESTA BRASIL. As florestas brasileiras. Disponível em: <http://www.florestabrasil.com.br/florplant.htm>. Acesso em: 25 julho 2001.

R. Árvore, Viçosa-MG, v.27, n.5, p.689-694, 2003 
LEITE, H. G. Conversão de troncos em multiprodutos de madeira, utilizando programação dinâmica. 1994. $230 \mathrm{f}$. Tese (Doutorado em Ciência Florestal) - Universidade Federal de Viçosa, Viçosa, 1994.

OLIVEIRA, A. D. et al. Avaliação econômica da vegetação de cerrado submetida a diferentes regimes de manejo e de povoamentos de eucalipto plantado em monocultivo.

Revista Cerne, v. 4, n. 1, p. 34-56, 1998.
REZENDE, J. L. P.; OLIVEIRA, A. D. Avaliação de projetos florestais. Viçosa, MG: UFV, Imprensa Universitária, 1993. 47 p.

SILVA, M. L.; FONTES, A. A.; LEITE, H. G. Rotação econômica em plantações de eucalipto não desbastadas e destinadas a multiprodutos. Revista Árvore, v. 23, n. 4, p. 403-412, 1999. 